\title{
ADENOME PARATHYROIDIEN INTRATHYMIQUE: A PROPOS D'UN CAS
}

\author{
A. SLAMA, T. TAYEB, M. GUEZGUEZ ${ }^{*}$, S. RAMMEH ${ }^{\star *}$, H. KOCHTALI \\ SERVICE DE CHIRURGIE MAXILLO-FACIALE \\ * SERVICE DE MÉDECINE NUCLÉAIRE \\ **SERVICE D'ANATOMIE PATHOLOGIQUE \\ C.H.U F.HACHED SOUSSE
}

\begin{abstract}
Introduction : Les adénomes parathyroïdiens posent le problème de leur diagnostic topographique. A partir d'un cas clinique d'adénome parathyroïdien intrathymique, les auteurs rappellent l'intérêt d'un bilan localisateur préopératoire.

Observation : II s'agit d'un homme âgé de 19 ans présentant une hyperparathyroïdie primitive. La scintigraphie à la Sestamibi a montré une hyperfixation inhabituelle du radiotraceur au niveau du creux sus sternal, permettant d'évoquer le diagnostic d'un adénome parathyroïdien intrathymique. Il a été réalisé une adénectomie en utilisant une voie d'abord cervicale et l'évolution post-opératoire était favorable.

Conclusion : La scintigraphie Sestamibi constitue l'examen de choix pour établir le diagnostic topographique des adénomes parathyroïdiens. Cet examen permet de limiter la voie d'abord chirurgicale et d'éviter les cervicotomies blanches. Mots-clés : Adénome parathyroïdien, thymus, scintigraphie.
\end{abstract}

Introduction : Parathyroïd adenomas present the difficulty of their exact topographic diagnosis. Through a clinical report of intrathymic parathyroïd adenoma, the authors insist on the importance of preoperative localization.

Observation : The authors present the case of a 19-year-old man with the diagnosis of primary hyperparathyroidism. The Sestamibi scintigraphy showed an abnormal hyperfixation in the sus sternal hollow. The diagnosis of an intrathymic parathyroïd adenoma was evoked. An adenectomy was performed using a cervical approach and postoperative outcomes were favourable.

Conclusion : The Sestamibi scintigraphy appears to be the best exam to establish topographic diagnosis of parathyroïd adenoma.

This exam permits to select the surgical approach and to avoid failed cervicotomy.

Keywords : Parathyroïd adenoma, thymus, scintigraphy.

\section{INTRODUCTION}

L'hyperparathyroïdie primaire (HPTp) résulte dans plus de $80 \%$ des cas d'un adénome, plus rarement d'une hyperplasie et exceptionnellement d'un carcinome parathyroïdien (1). Dans la majorité des cas, l'adénome se développe au niveau des glandes parathyroïdiennes inférieures et sa localisation intrathymique est rare $(2,3)$. A travers une observation clinique, les auteurs rappellent la nécessité d'un bilan localisateur des adénomes parathyroïdiens intrathymiques.

\section{OBSERVATIONS}

II s'agit d'un homme âgé de 19 ans, sans antécédents pathologiques, qui a été hospitalisé pour une lithiase rénale gauche associée à une hypercalcémie menaçante. L'examen cervicofacial était pauvre. En effet, la palpation n'a pas retrouvé de nodules dans la loge thyroïdienne ni d'adénopathies cervicales. L'examen général était sans particularités avec absence de signes d'insuffisance réna- le chronique.

Le bilan biologique a montré une hypercalcémie à 3,56 $\mathrm{mmol} / \mathrm{l}$ (valeurs normales 2,38-2,70 $\mathrm{mmol} / \mathrm{l}$ ), une hypophosphorémie à $0,53 \mathrm{mmol} / \mathrm{l}$ (valeurs normales $0,87-1,45$ $\mathrm{mmol} / \mathrm{l})$, une hyperparathormonémie à $190,2 \mathrm{pg} / \mathrm{ml}$ (valeurs normales $15-65 \mathrm{pg} / \mathrm{ml}$ ) et une fonction rénale normale. Devant ces signes biologiques, le diagnostic d'HPTp a été retenu.

Le bilan localisateur de la lésion parathyroïdienne a comporté une scintigraphie à la Sestamibi qui a révélé une hyperfixation inhabituelle du radiotraceur au niveau du creux sus sternal dans le prolongement et à distance du lobe thyroïdien gauche (Fig. 1). II n'a pas été noté d'autres foyers hyperfixants ni au niveau de la région cervicale ni au niveau de la région thoracique.

Cet aspect scintigraphique était fortement évocateur d'un foyer parathyroïdien intrathymique et hypersécrétant. 


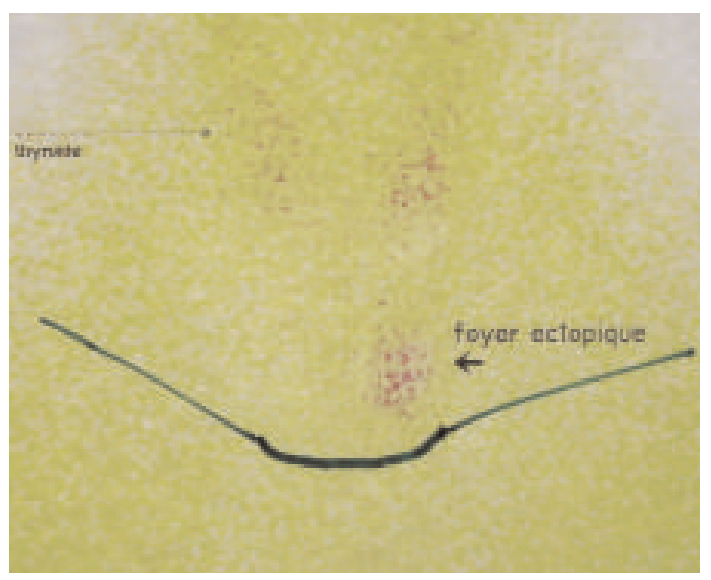

Fig. 1: Scintigraphie Sestamibi montrant une hyperfixation au niveau du creux sus sternal.

Après un abord cervical limité du creux sus sternal, on a découvert dans le prolongement cervical du thymus une lésion ovalaire, bien limitée, d'aspect rouge violacé et de $10 \mathrm{~mm}$ de grand axe (Fig. 2). II a été réalisé une exérèse de la lésion emportant le tissu thymique avoisinant (Fig. 3).

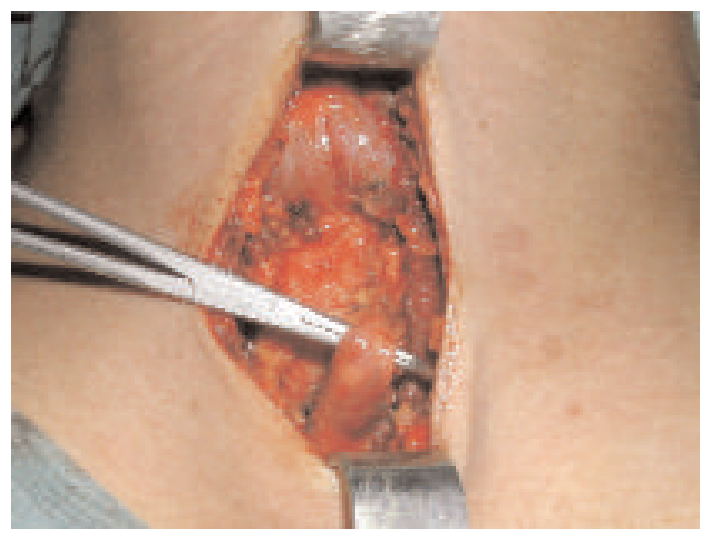

Fig. 2: Aspect per-opératoire de l'adénome parathyroïdien intrathymique.

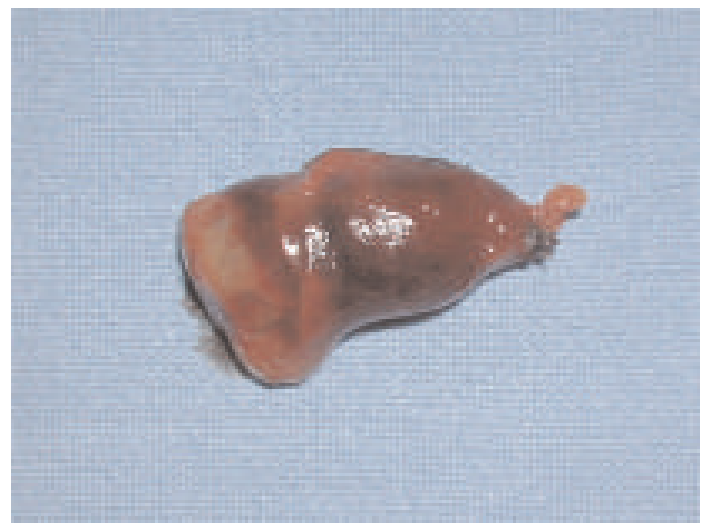

Fig.3: Pièce d'exérèse emportant l'adénome et le tissu thymique avoisinant.
L'examen anatomopathologique de la pièce d'exérèse a conclut au diagnostic d'adénome parathyroïdien intrathymique (Fig. 4).

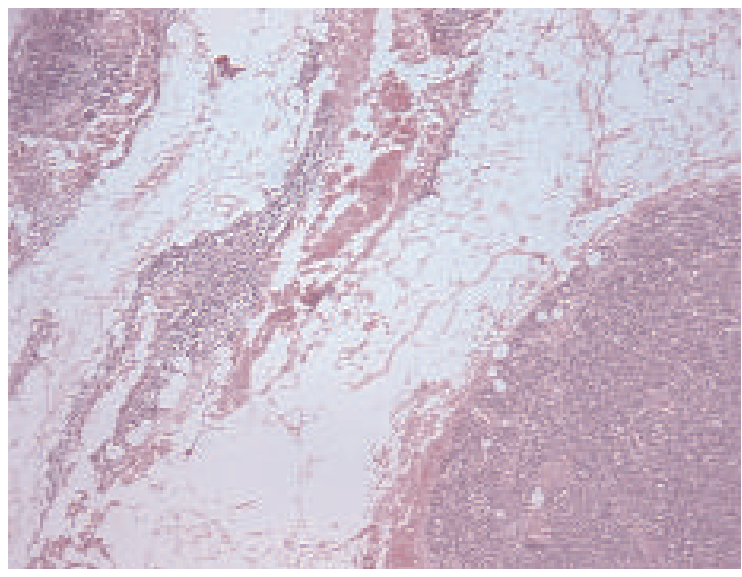

Fig. 4: (HEx50): Aspect histologique de l'adénome parathyroïdien (en bas et à droite)

inclus dans le tissu thymique (en haut et à gauche).

L'évolution postopératoire était favorable avec une normalisation des taux de la calcémie, de la phosphorémie et de la parathormonémie. Après un recul de 6 mois, il n'a pas été noté de récidives.

\section{DISCUSSION}

L'HPTp est secondaire, dans 80 à $85 \%$ des cas, à un adénome parathyroïdien qui siège essentiellement au niveau des glandes parathyroïdes inférieures $(1,2)$. L'incidence des adénomes parathyrö̈diens intrathymiques est variable selon les auteurs $(3,4)$.

Dans une série de 231 patients opérés pour des hyperparathyroïdies, Phitayakorn et McHenry ont retrouvé 37 cas (soit $16 \%$ des cas) de lésions ectopiques dont 7 cas intrathymiques (soit 3\% des cas) (5). Selon l'étude de Dhouib, $88 \%$ des adénomes parathyrö̈diens siègeraient au niveau des glandes inférieures et un seul cas d'adénome intrathymique a été constaté parmi les 34 patients opérés pour HPTp (6).

Le recours à un bilan localisateur des lésions parathyroïdiennes hypersécrétantes, surtout infraclinique, est actuellement fortement recommandé en préopératoire(7). En effet, La localisation préopératoire de l'adénome parathyroïdien permet de limiter la voie d'abord chirurgicale, de raccourcir le temps opératoire et d'éviter les hypocalcémies postopératoires observées en cas d'exploration systématique des 4 sites habituels des parathyroïdes. En cas d'adénome ectopique ou intrathymique, le bilan localisateur est impératif puisqu'il permet d'éviter la cervicotomie «blanche » (8).

A coté de l'examen clinique, l'imagerie constitue un examen indispensable pour le diagnostic positif et topographique de l'adénome parathyroïdien. 
L'échographie parathyroïdienne, l'examen le plus prescrit, reste opérateur dépendant avec une sensibilité variable entre 55 et $85 \%(9,10)$.

La tomodensitométrie est surtout indiquée en cas de négativité de l'échographie ou avant une reprise chirurgicale pour des hyperparathyroïdies persistantes ou récidivantes (11).

La scintigraphie de soustraction au 99mTc-Sestamibi est actuellement considérée comme la technique de choix puisqu'elle présente une sensibilité de l'ordre de $95 \%$ et offre une meilleure qualité d'image que la tomodensitométrie avec une irradiation plus faible $(11,12)$. D'après Mitchell, cet examen a permis d'identifier 16 adénomes parmi 17 cas et 19 hyperplasies glandulaires parmi 21 cas (13).

Chez notre patient, la scintigraphie à la Sestamibi a permis de faire le diagnostic topographique de l'adénome et d'en faciliter le traitement chirurgical en évitant la cervicotomie « blanche » et l'hypocalcémie postopératoire.

\section{CONCLUSION}

Le diagnostic topographique des adénomes parathyroïdiens intrathymiques, qui repose essentiellement sur la scintigraphie Sestamibi, permet d'éviter les hypocalcémies postopératoires et les cervicotomies «blanche».

\section{REFERENCES}

1- Ruda JM, Hollenbeak CS, Stack BC. A systematic review of the diagnosis and treatment of primary hyperparathyroidism from 1995 to 2003. Otolaryngol Head Neck Surg. 2005; 132: 359-72.

2- Wang CA. The anatomical basis of parathyroid surgery. Ann Surg.1976; 183:271-75.

3- Peeler B.B, Martin W.H, Sandler M.P. Sestamibi parathyroid scanning and preoperative localization studies for patients with recurrent/persistent hyperparathyroidism: development of an optimal localization strategy. Am Surg. 1997; 63:37- 46 .

4- Hamaloglu E, Fani Bozkurt M, Dogan R. Unexpected Detection of an Intrathymic Parathyroid Adenoma by Tc-99m-MIBI Scintigraphy: The role of scintigraphy in planning surgical treatment. The Endocrinologist. 2005; 15: 366-69. 5- Phitayakorn R, McHenry C.R. Incidence and location of ectopic abnormal parathyroid glands. Ann Surg. 2006; 191: 418- 23.

6- Dhouib H, Hammami B, Ben Thabet A, Drira M.M. Traitement chirurgical de I'hyperparathyroïdie primaire: techniques et résultats. J.Tun ORL. 2006; 16: 8-11

7- Boggs J.E, Irvin G.L, Carnerio D.M. The evolution of parathyroïdectomy fai- lures. Surgery.1999; 126:998-1003.

8- Bilezikian JP, Potts JT, Fuleihan G.H. Summary statement from a workshop on asymptomatic primary hyperparathyroidism: a perspective for the 21 st century. J.Clin Endocrinol Metab. 2002; 87: 5353- 61.

9- Chou F.F, Wang P.W, Sheen-Chen S.M. Preoperative localisation of parathyroid glands in primary hyperparathyroidism. Eur J Surg. 1997; 163: 889-95.

10- Jeanguillaume $C$, Hindie $E$, Mellière $D$. Récents progrès en imagerie scintigraphiques des parathyroides. Ann Endocrinol. 1997 ; 58: 143-51.

11- Ahuja A.T, Wong, K.Y, Ching A.S.C. Imaging for primary hyperparathyroidism- What beginners should know. Clinical Radiology. 2004; 59: 967-76.

12- Mullan BP. Nuclear medicine imaging of the parathyroid [review]. Otolaryngol Clin North Am. 2004; 37: 909-29.

13- D'Avanzo A, Parangi S, Morita E. False positive 99mTc Sestamibi scans in patients with osteitis fibrosa cystica and brown tumours. Eur J Surg. 2001; 167:592-97 